\title{
Real Time In-situ Detection System Research for Methane in Offshore Shallow Gas based on Thin Film Interface
}

\author{
Miao Cheng-xing ${ }^{1}$, Li Qing ${ }^{1, a}$ and Jia Sheng-yao ${ }^{1}$ \\ ${ }^{1}$ China Jiliang University, Institute of mechanical and electrical engineering, Hangzhou 310018 ,China
}

\begin{abstract}
In order to get ridded of the non real-time detection methods of artificial site sampled and laboratory instrument analyzed in the field of methane detection in the offshore shallow gas, real-time in-situ detection system for methane in offshore shallow gas was designed by the film interface.The methane in the offshore shallow gas through the gas-liquid separation membrane of polymer permeation into the system internal detection probe, analog infrared micro gas sensor sensed the methane concentration and the corresponded output value, data acquisition and communication node fitted into standard gas concentration.Based on the experimental data compared with the traditional detection method, and further analyzed the causes of error produced by the case experiment. The application results show that the system can achieve a single borehole layout, long-term on-line in-situ on-line detection, and improve the detection efficiency and the timeliness of the detection data.
\end{abstract}

\section{Introduction}

Shallow marine gas was widely distributed in the Gulf near the Yangtze River Delta and Hangzhou Bay area. On the one hand, shallow gas in the sea can be used as a new energy utilization and development; On the other hand, the leakage and eruption of shallow gas in the offshore area will cause great harm to the construction of marine engineering. Therefore, the real-time in-situ on-line detection method for offshore shallow gas has become an urgent need for marine energy development and engineering construction in China.

At present, the method of methane detection in offshore shallow gas was mainly used as exploration drilling method. The method first obtains the location of shallow gas enrichment by means of geophysical exploration, and then separate the shallow gas and sea water from the laboratory conditions, then the gas concentration information of shallow gas was measured by gas chromatography.

With the development of modern science and technology, some real time in-situ detection methods have been applied to the detection of shallow gas in the bottom of the sea. A new geological environment, pore pressure static sounding device, developed by Holland Hui $\mathrm{Gu}$ company, has been installed on the static sounding device with a film interface detector. The glass fiber membrane of water breathable function. With the help of the mother ship, the device can detect the methane concentration in the shallow layer of the seabed to a certain extent. However, each test must be penetrated into the seabed by the mother ship, which can not be carried out once and in a long time in situ continuous detection; moreover, the instrument was imported from abroad at present. All of these results increased the cost of real-time in-situ detection of methane in offshore shallow gas.

On the basis of film interface detection, this paper change the selection of die body, and designed a real-time in situ methane detection system in the offshore shallow gas by used the micro infrared gas sensor. The key technology was that the detection system can provide a water-proof work environment for gas sensors. Methane in shallow gas can be penetrated into the detection system through multilayer nanomomm, and the detection capacity of methane concentration in $0-100 \%$ wide range gas by gas sensor. It is possible to achieve long-term monitoring of the escape of methane in shallow gas.

\section{Design of detection system}

\subsection{System overall plan}

A real-time in-situ detection system for methane in offshore shallow gas based on the film interface, it was composed of five part. The permeable stone that filters the sediment; the gas separation probe that separate methane from the sea and detect the methane in the shallow gas. It was as specific as Figure 1. 


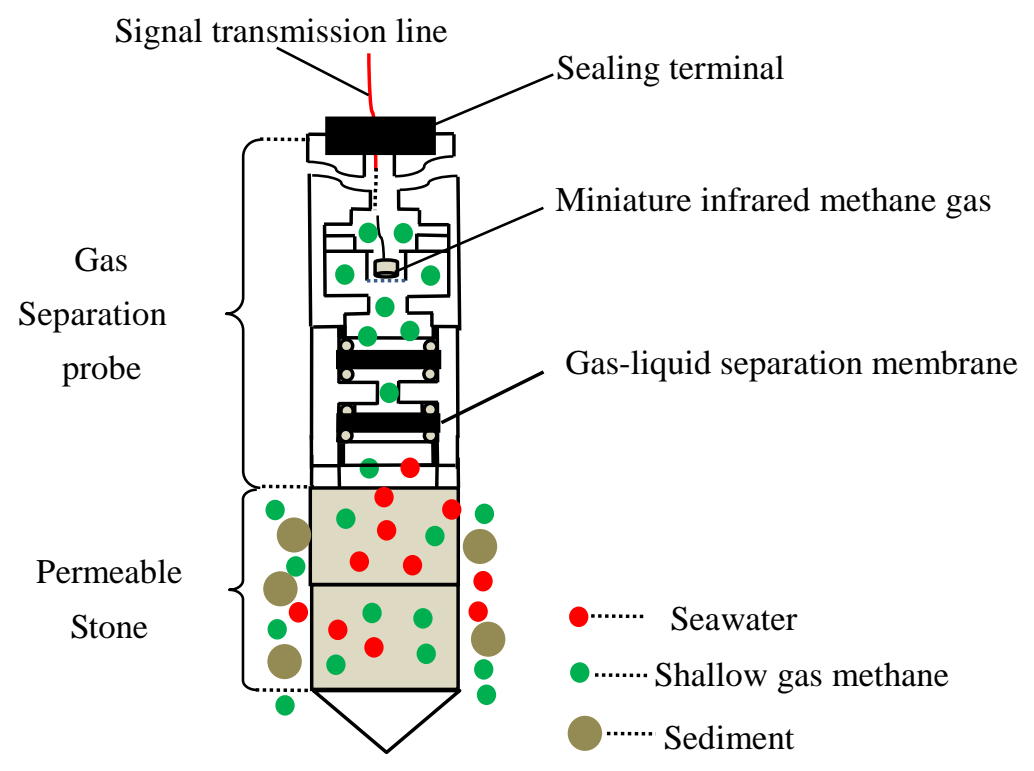

Figure 1. Methane detection process in shallow gas

The data acquisition and communication node obtained the analog signal output from the micro infrared gas sensor through the serial port protocol, and converted it into the standard methane concentration value, and send the concentration value to the marine carrier by wireless transmission. It was as specific as Figure 2.

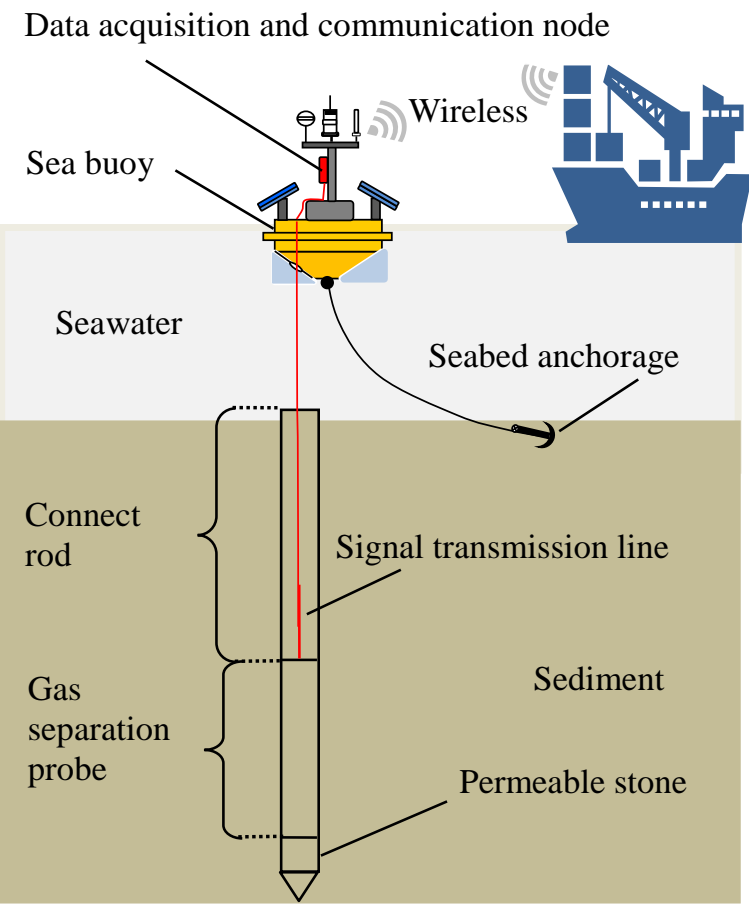

Figure 2. Overall plan design

\subsection{Design of methane collection system in shallow gas}

\subsubsection{Gas-liquid separation membrane}

In the design the gas-liquid separation membrane was a multilayer nanomomm made of poly two methyl siloxane material, and it was also a biomimetic super hydrophobic membrane. The semi organic and semi inorganic structural properties made it highly affinity to volatile organic compounds and dissolved gases, and have high block properties for water and salt and other inorganic substances. The separation and osmosis principle of the membrane was illustrated in Figure 3. 


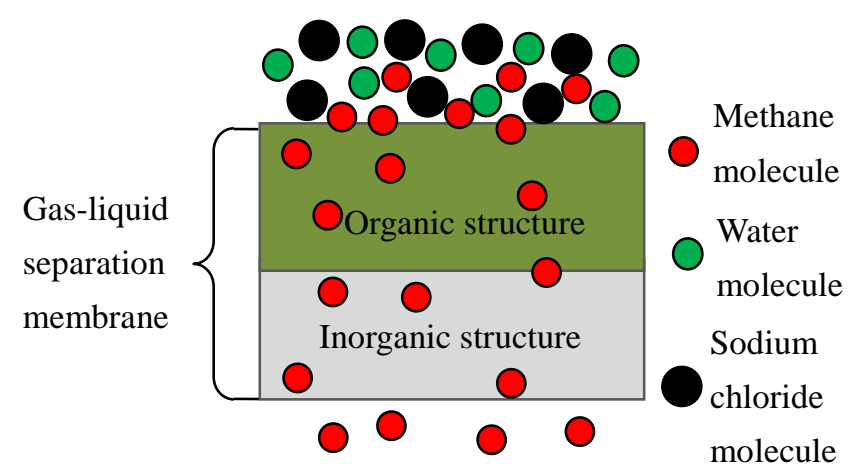

Figure 3. Principle of membrane separation and permeation

In order to verified the permeability and compression ability of the membrane, a test model was established, as shown in Figure 4, and the gas flow velocity was simulated for the model, as shown in Figure 5.

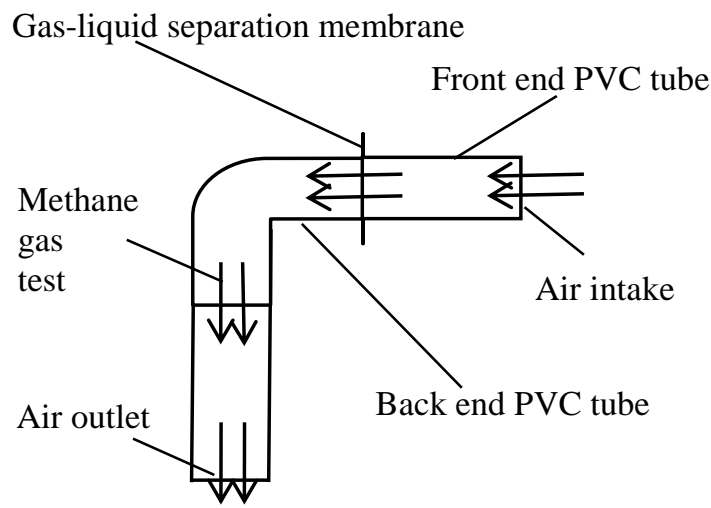

Figure 4. Permeability compression model of membrane body

In the test model, the gas flow valve was controlled by the gas flow valve to tested the methane gas flow from the model inlet to the interior of the model. The monolayer gas liquid separation membrane was installed at the distance of $50 \mathrm{~cm}$ from the air inlet, and the front end PVC tube and the back end PVC tube were tightly meshed, and the surface of the die body was tightened and the gas was fully exposed to the surface of the die body. Applied sealant to the mesh to prevent leakage of test gas. Thus, a gas flow velocity model of a methane test gas which must pass through a tight gas liquid separation membrane from the intake and released from the outlet is formed.

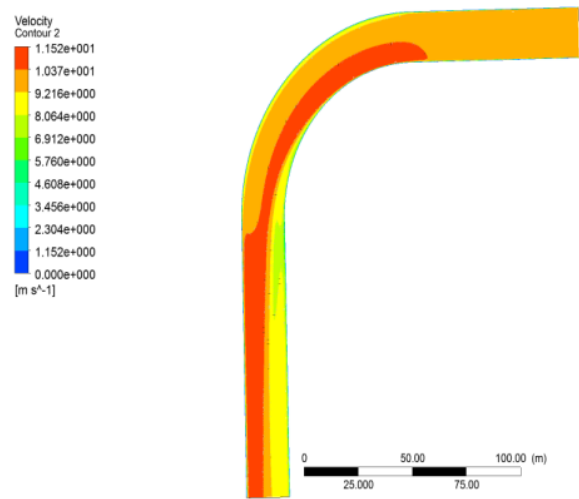

Figure 5. Simulation of model gas flow velocity
It can be seen from Figure 5 that there was no change in the velocity of methane gas before and after pass through the membrane. This indicated that the membrane has good permeability to methane gas.

\subsubsection{Gas separation probe}

The gas separation probe was the key component to realize the separation of methane from shallow water and realize the detection. Its components include: water gas collection room, compact gasket, gas-liquid separation room, sensor placement ent room. It was as specific as Figure 6.

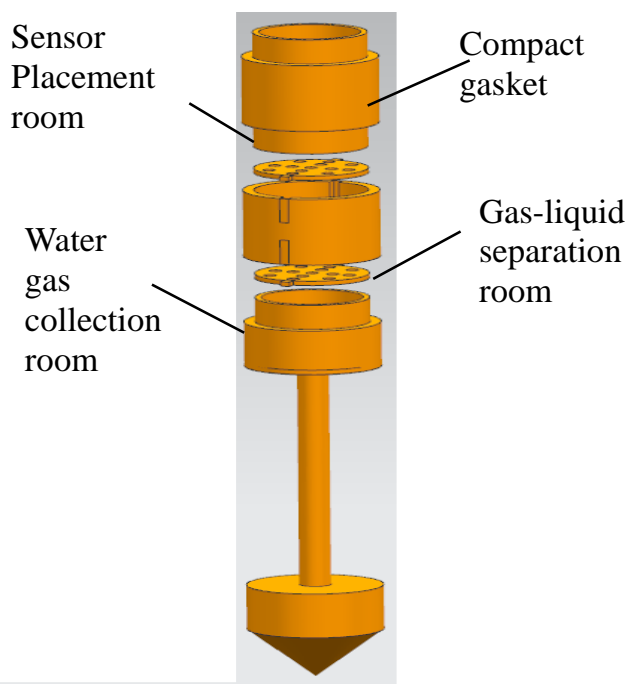

Figure 6. Probe composition structure

A rubber sealing washer with certain specifications was fitted between the various components. The sealing terminal at the upper end of the probe was a waterproof seal with a thread, which can be attached to the probe through a sealing head and put into a signal lead in the tube. Thus, the full sealing property of the gas separation probe was realized, and the safe and waterproof work environment for the gas sensor was provided. The concrete objects were as shown in Figure 7. 


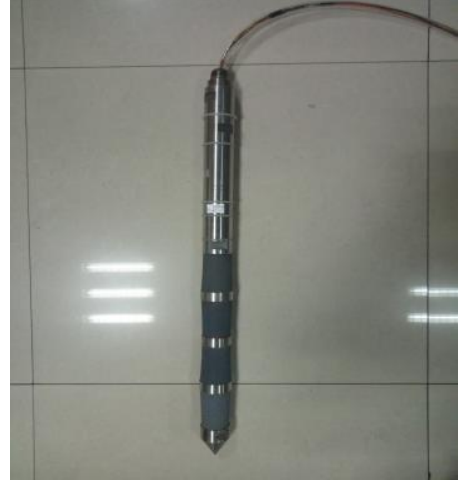

Figure 7. Probe concrete object

\subsection{Design of methane detection system in shallow gas}

According to the traditional artificial field sampling and laboratory instrument analysis, the composition of the shallow gas in the offshore was mainly composed of high methane gas, which was usually more than $70 \%$ and contains a small amount of other inert gases. Therefore, in the system design, the gas sensor was required to have a wide range of methane concentration detection ability, and the volume was small enough to be installed inside the probe, and the detection process was safe and reliable.

In the design, the oxide semiconductor methane gas sensor, the catalytic combustion methane gas sensor and the micro infrared methane gas sensor are selected respectively. First, the initial values of the selected three kinds of methane gas sensors were tested. The results were Figure 8 and Figure 9.

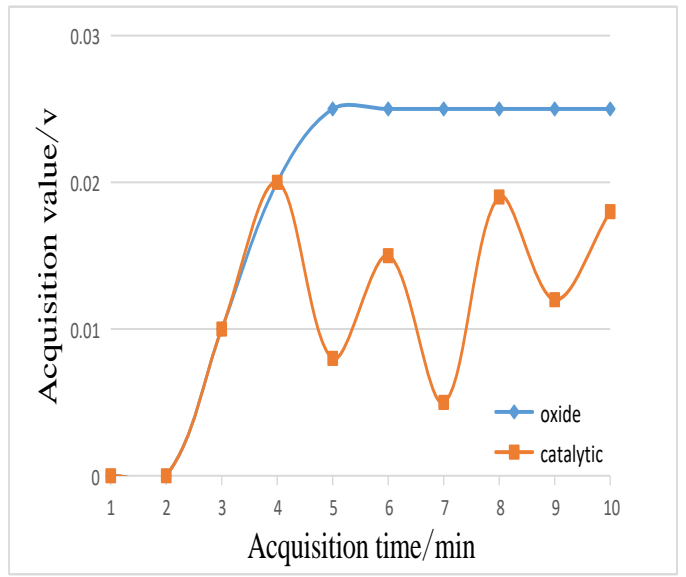

Figure 8. Initial test results of traditional methane gas sensor

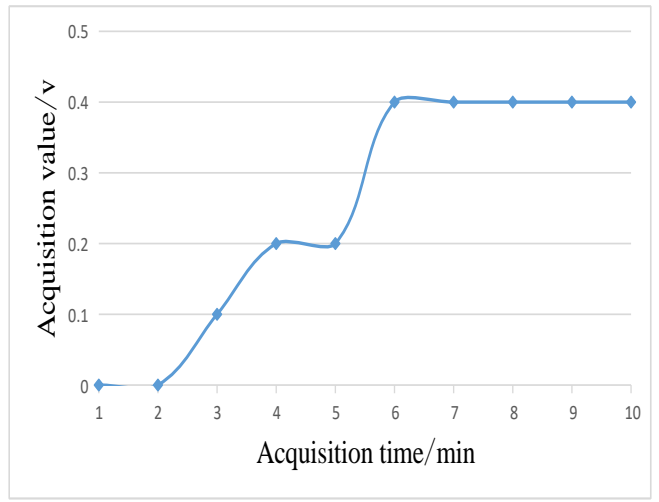

Figure 9. Initial value test result of micro infrared gas sensor

It can be seen from the above diagram that the oxide semiconductor and micro infrared methane sensor have good test results. The following two gas sensors were selected in the same sealed container, and the methane gas was tested at different concentrations. The simulated output test results of the two sensors were shown in Figure 10 and Figure 11 .

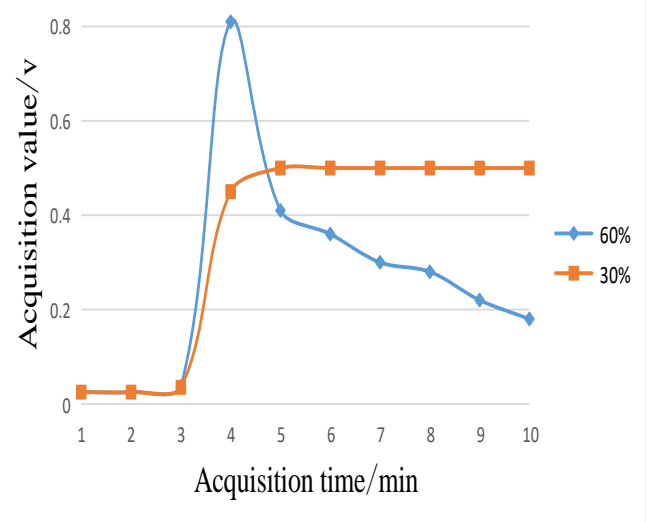

Figure 10. Measurement results of methane sensor of oxide semiconductor

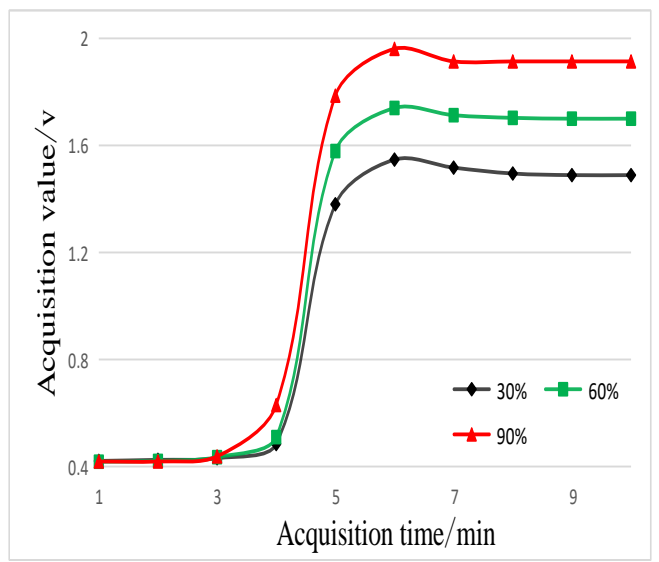

Figure 11. Measurement results of micro infrared methane gas sensor

It can be seen from the diagram that the micro infrared methane gas sensor has more stable analog output and the output amplitude was more significant after the methane test gas of different concentrations was passed through. 
The Hydro C micro infrared methane gas sensor developed by Contros company in Britain was a typical representative of methane detection in the field of industry at present, with a height of $10 \mathrm{~cm}$ and $20 \mathrm{~cm}$ in diameter. Therefore, the selection of micro infrared methane gas sensor in the system design was quite in line with the design requirements.
In order to fund out whether the system has the ability to accurately measure methane in shallow gas in the seabed sediments, a simulated test scheme was designed under laboratory conditions. The specific operation was as shown in Figure 12.

\section{Simulation experiment and data fitting}

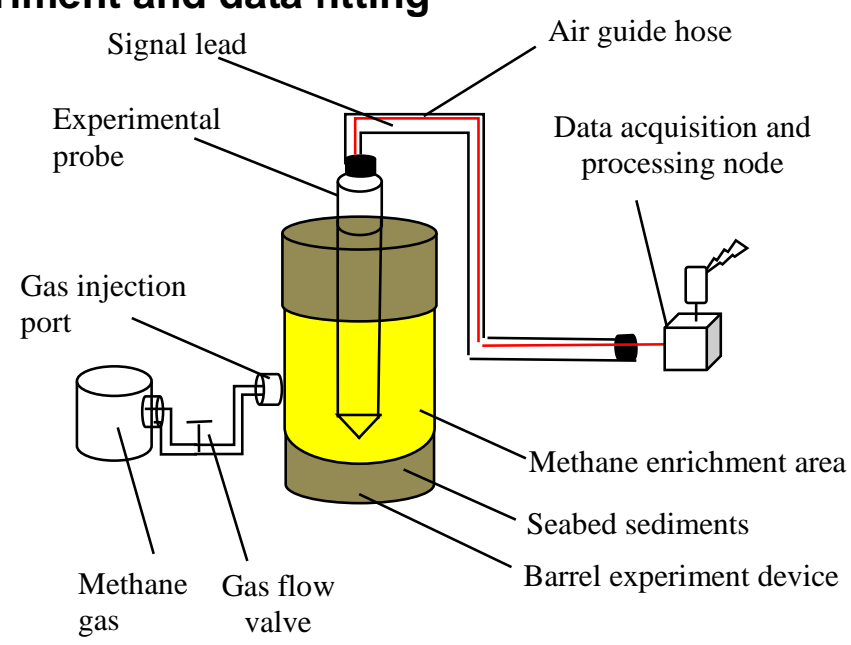

Figure 12. Simulation experiment implementation

In the barrel experiment device, acquired sediment is added to the device before, and the methane gas of the specific concentration was injected into the gas injection port through the gas injection port, which was reserved by the barrel experiment device, so that the methane enrichment area was formed in the sediment in the device, so that the methane in the enrichment area was fully mixed and closer to the real shallow gas reservoir, Methane inlet gas pipes are equipped with gas flow valves to control the speed and duration of methane input. The assembled experimental probe is inserted into the barrel device through the reserved port at the upper end of the device, and the reserved port was sealed. The test data was transmitted to the host computer by wireless transmission, and displayed and recorded.

The experimental gases with different concentrations of methane were tested respectively. The results were shown in Table 1.

Table 1. Experimental gas simulation results of methane at different concentrations

\begin{tabular}{cc}
\hline $\begin{array}{c}\text { Experimental gas } \\
\text { concentration value/(\%) }\end{array}$ & Output simulation/(V) \\
\hline 1 & 1.210 \\
10 & 1.306 \\
20 & 1.406 \\
30 & 1.477 \\
40 & 1.587 \\
50 & 1.667 \\
60 & 1.687 \\
70 & 1.807 \\
\hline
\end{tabular}

\begin{tabular}{ll}
\hline 80 & 1.878 \\
90 & 1.887 \\
\hline
\end{tabular}

According to the basic idea of least square method, a reflection function was constructed:

$$
\mathrm{g}_{m}(x)=a_{0}+a_{1} x+\ldots . .+a_{m} x^{m}
$$

The mathematical relationship between experimental gas concentration and output simulation was obtained from the solution of linear equation:

$$
\mathrm{g}(x)=\left(-3.3 \times 10^{-5}\right) x^{2}+0.011 x+1.2
$$

Among them, $\mathrm{g}(x)$ is the analog output of micro infrared gas sensor, $\mathrm{V} ; \boldsymbol{x}$ is the experimental gas concentration value, $\%$.

The relationship between experimental gas concentration and output simulation was directly expressed by used MatLab. It was as specific as Figure 13.

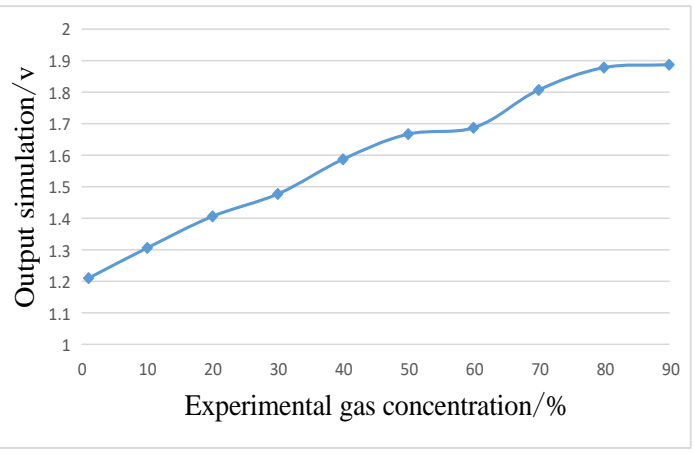

Figure 13. Data fitting curve 
Parallel experiments were carried out to obtain the concentration values of methane at different concentrations, as shown in Table 2.

Table 2. Experimental gas concentration results of different concentrations of methane

\begin{tabular}{lcc}
\hline $\begin{array}{l}\text { Experimental } \\
\text { concentration/ } / \%)\end{array}$ & $\begin{array}{l}\text { Result } \\
\text { concentration/ } \\
(\%)\end{array}$ & Error/ (\%) \\
\hline 1 & 0.1 & -0.9 \\
10 & 8.2 & -1.8 \\
20 & 17.3 & -2.7 \\
30 & 27.6 & -2.4 \\
40 & 38.1 & -1.9 \\
50 & 48.0 & -2.0 \\
60 & 58.4 & -1.6 \\
70 & 68.9 & -1.1 \\
80 & 79.2 & -0.8 \\
90 & 89.4 & -0.6 \\
\hline
\end{tabular}

It can be seen from the above table that there was a certain error between the obtained results and the actual experimental gas concentration, and it was generally lower than the actual experimental gas concentration value, and the maximum error was $-2.7 \%$. However, when the actual experimental gas concentration was larger, this error will decrease. The possible reasons are: (1) The error of mathematical calculation model caused by data fitting curve. (2) The simulated methane in the seabed sediments was less, resulting in uneven gas distribution in the methane enriched area and less gas entering the detection system.

\section{Experimental and discussion}

According to the experimental results, the following measures were made: (1)The gas flow valve was used to increased the flow of the experimental gas into the device and prolong the gas injection time; (2)According to the fitting curve analysis between the experimental gas concentration and the output simulation, it can be seen that the different experimental gas concentration intervals correspond to the mathematical models with different output analog quantities; therefore, if the interval fitting between the real gas concentration and the output simulation may decrease the result concentration, the result concentration may be reduced. The error between the model and the experimental gas concentration was calculated.

The following data were piecewise fitted. The mathematical relationship was as follows:

(1) $x \in[60,90]$,

$\mathrm{g}(x)=\left(-2.8 \times 10^{-4}\right) x^{2}+0.048 x-0.21$

(2) $x \in[30,50]$,

$\mathrm{g}(x)=\left(-1.5 \times 10^{-4}\right) x^{2}+0.021 x+0.97$
(3) $x \in[1,20]$,

$$
\mathrm{g}(x)=\left(-3.5 \times 10^{-5}\right) x^{2}+0.011 x+1.2
$$

The piecewise fitted function was used to carry out parallel experiments, and the results were shown in Table 3.

Table 3. Experimental gas concentration results of different concentrations of methane

\begin{tabular}{ccc}
\hline $\begin{array}{l}\text { Experimental gas } \\
\text { concentration/ } \\
(\%)\end{array}$ & $\begin{array}{l}\text { Result } \\
\text { concentration/ } \\
(\%)\end{array}$ & Error/ $(\%)$ \\
\hline 1 & 0.5 & -0.5 \\
10 & 9.0 & -1.0 \\
20 & 18.6 & -1.4 \\
30 & 28.6 & -1.4 \\
40 & 39.1 & -0.9 \\
50 & 49.1 & -0.9 \\
60 & 59.2 & -0.8 \\
70 & 69.5 & -0.5 \\
80 & 79.5 & -0.5 \\
90 & 89.6 & -0.4 \\
\hline
\end{tabular}

Although there was still a certain error in the numerical results obtained by the experiment, compared with the test result table 3 obtained before the fitting function, it can be seen that the piecewise fitting function reduced the model fitting calculation error between the results obtained by the simulation experiment and the actual experimental gas concentration.

\section{Conclusion and Prospect}

(1)The results of simulation experiments under laboratory conditions showed that methane in shallow gas can be penetrated into the detection probe of the multilayer nanomomm film. The gas separation probe provided a waterproof and reliable work environment for the gas sensor, and then the wide range detection of methane in the diffused shallow gas was realized with the micro infrared methane gas sensor.

(2)The system can effectively real-time in situ on-line detection of methane in shallow gas in the offshore sediments, and can transmit the methane concentration in the shallow gas enrichment area to the upper computer in real time. The line monitoring of methane injection in shallow gas. The test cost was reduced, the detection cycle was shortened, and the operability was strong.

(3)In this paper, based on thin film interface detection, we only studied methane detection in shallow gas with a certain degree of singleness. In the future, we can expand other functions on this basis: detecting carbon dioxide concentration; exploring the origin of gas in shallow gas and gas migration in gas reservoirs. 


\section{Acknowledgment}

Zhejiang provincial key research project (2018C03031) National Natural Science Foundation of China (41376111)

State Administration of quality supervision, inspection and Quarantine General Plan of science and Technology (2017QK053)

\section{References}

1. Xing Lei, Jiao Jingjuan, Liu Xueqin, et al. Analysis of shallow gas distribution and seismic characteristics in Bohai sea area. Journal of Ocean University of China: Natural Science Edition, 2017(11):70-78

2. Zhou Yinkang, Yan Changhong, Li Xueqian, et al. Spatial distribution of shallow gas in the Quaternary Holocene soft soil and its disaster control characteristics. Engineering Investigation, 2016, 44(5):17-20

3. Chen Zhongxuan, Lai Xianghua, Liao Linyan, et al. Shallow gas detection method based on MIP-CPT Technology: a case study of Zhoushan sea area in East China Sea. Journal of petroleum, 2016, 37(2):207-213

4. Zhou Bo, Yang Jin, Zhang Bailing, et al. Prediction and control technology for shallow geological disasters in deep water. Frontiers of Marine Geology, 2012(1):51-54

5. Zhu Yu. Application of thin film interface detector in contaminated site investigation. Urban Roads Bridges \& Flood Control, 2015(6):228-231

6. Li Jie, Qiu Yafeng, Qian Yunsheng, et al. Design and analysis of glass fiber production system based on MCP. Applied Optics, 2018(1):112-116

7. Xue Zhe. Study on the preparation and application of multi-layer nanostructured membrane. Nanjing University of Technology, 2015

8. [8] Cui Xuyuan, Gao Wanxian. Research status and development prospect of biomimetic super hydrophobic materials. Knitting industry, 2018(1):63-67

9. Ming-Ji S U, Ping J I, Wang Z G, et al. Research Progress of Biomimetic Superhydrophobic Surfaces. Chinese Journal of Colloid \& Polymer, 2016

10. $\mathrm{Yu} \mathrm{H} \mathrm{B,} \mathrm{Li} \mathrm{R} \mathrm{F.} \mathrm{Preparation} \mathrm{and} \mathrm{properties} \mathrm{of}$ biomimetic superhydrophobic composite coating. Surface Engineering, 2016, 32(2):79-84

11. Hong Wensheng, Deng Yu, Huang Zhigang, et al. Preparation of poly two methylsiloxane super hydrophobic surface by nanosecond ultraviolet laser. Polymer materials science and Engineering, 2018(1):125-129

12. Wang Jianming. The physical meaning and calculation method of Lambert Beer law. Journal of Higher Continuing Education, 2000(3):32-33

13. Huang Bing, Wang Ying, Wang Qiang, et al. Shallow gas detection based on ARM. Mechanical and electrical engineering, 2015, 32(7):915-919
14. [14] Fonollosa J, Rubio R, Hartwig S, et al. Design and fabrication of silicon-based mid infrared multi-lenses for gas sensing applications. Sensors \& Actuators B Chemical, 2008, 132(2):498-507

15. Anderson, A.L., Hampton, L.D. Acoustics of gas-bearing sediments II. Measurements and models. The Journal of the Acoustical Society of America, 1980, 67(6): 1890-1903

16. Hiu Bin, Li Wei, Liu Haijun, et al. Research progress of micro nano structure two tin oxide gas sensor. Application of chemical industry, 2016, 45(11):2139-2143

17. Liu Zengwei, Yang Xi, Sun Jie, et al. Research progress of two oxidizing tin based gas sensitive materials. Material guide, 2017, 31(s1):23-29

18. Xie Peng, Zhang Hongmei. Design and calculation analysis of new sensor based on infrared wavelength. Instrument technology and sensor, 2016(7):26-28

19. Liu Yanxiang, Li Tie, Yao Jinzhi, et al. Micro infrared methane gas sensor for drilling detection. Laser and infrared, 2017, 47(9):1137-1141

20. Ren Weiqi, Yang Zhizhou. Research on portable gas detection system based on $\mathrm{CH} 4$ infrared gas sensor. Engineering Technology: Abstract Edition, 2016(6):00095-00095 